\title{
Extremal chemical trees of the first reverse Zagreb beta index
}

\author{
Süleyman Ediz and Mesut Semiz \\ Faculty of Education, Yüzüncü Y1l University \\ Van, Turkey \\ e-mails: suleymanediz@yyu. edu.tr, \\ mesutsemiz@gmail.com
}

Received: 10 October 2017 Revised: 13 September $2018 \quad$ Accepted: 11 October 2018

\begin{abstract}
The reverse vertex degree of a vertex $v$ of a simple connected graph $G$ defined as $c_{v}=\Delta-d_{v}+1$, where $\Delta$ denotes the largest of all degrees of vertices of $G$ and $d_{v}$ denotes the number of edges incident to $v$. The first reverse Zagreb beta index of a simple connected graph $G$ defined as $C M_{1}^{\beta}(G)=\sum_{u v \in E(G)}\left(c_{u}+c_{v}\right)$. In this paper, we characterized maximum chemical trees with respect to the first reverse Zagreb beta index.
\end{abstract}

Keywords: Zagreb indices, Reverse Zagreb indices.

2010 Mathematics Subject Classification: $05 \mathrm{C} 07$.

\section{Introduction}

A topological index, which is a graph invariant, i.e., it does not depend on the labeling or pictorial representation of the graph, is a numerical parameter mathematically derived from the graph structure. The topological indices of molecular graphs are widely used for establishing correlations between the structure of a molecular compound and its physico-chemical properties or biological activity. These indices are used in quantitive structure property relations (QSPR) research. The first distance based topological index was proposed by Wiener in 1947 for modeling the physical properties of alcanes [11], and after him, hundred topological indices were defined by chemists and mathematicians and so many properties of chemical structures were studied. 
More than forty years ago Gutman and Trinajstić defined Zagreb indices, which are degree based topological indices [8]. These topological indices were proposed to be measures of branching of the carbon-atom skeleton in [7]. The first and second Zagreb indices of a simple connected graph $G$ defined as follows

$$
M_{1}(G)=\sum_{u \in V(G)} d_{u}^{2}
$$

and

$$
M_{2}(G)=\sum_{u v \in E(G)} d_{u} d_{v}
$$

where $u, V(G), u v$ and $E(G)$ denote a vertex of $G$, the vertex set of $G$, an edge of $G$ and the edge set of $G$, respectively. For details of the chemical applications and the mathematical theory of the Zagreb indices, see the surveys $[1,6,9,10]$. For the another version of the first Zagreb index,

$$
M_{1}(G)=\sum_{u v \in E(G)}\left(d_{u}+d_{v}\right)
$$

see in [10].

Recently, reverse vertex degree of a vertex and reverse Zagreb indices of a simple connected graph have been defined in [2]. The reverse vertex degree of a vertex $v$ of a simple connected graph $G$ defined as

$$
c_{v}=\Delta-d_{v}+1
$$

where $\Delta$ denotes the largest of all degrees of vertices of $G$ and $d_{v}$ denotes the number of edges incident to $v$. The total reverse vertex degree defined as

$$
T R(G)=\sum_{u \in V(G)} c_{u} .
$$

The first reverse Zagreb alpha index of a simple connected graph $G$ defined as

$$
C M_{1}^{\alpha}(G)=\sum_{u \in V(G)} c_{u}^{2}
$$

The first reverse Zagreb beta index of a simple connected graph $G$ defined as

$$
C M_{1}^{\beta}(G)=\sum_{u v \in E(G)}\left(c_{u}+c_{v}\right) .
$$

And the second reverse Zagreb index of a simple connected graph $G$ defined as

$$
C M_{2}(G)=\sum_{u v \in E(G)} c_{u} c_{v}
$$

Maximum chemical trees of the second Zagreb index were characterized in [2]. The reverse Zagreb indices of Cartesian product of two graphs were computed in [5]. Maximal graphs with respect to the first reverse Zagreb beta index have been determined in [4]. Extremal chemical trees of the first reverse Zagreb alpha index have been determined in [3].

The aim of this paper is to characterize maximum chemical trees with respect to the first reverse Zagreb beta index. 


\section{Maximum chemical trees with respect to the first reverse Zagreb beta index}

In this section, our goal is to obtain sharp upper bound on the first reverse Zagreb beta index of chemical trees and, accordingly, to characterize the chemical trees with maximal values of the first reverse Zagreb beta index.

Lemma 2.1. If $G$ is a chemical tree with at least one vertex of degree 3, then its first reverse Zagreb beta index cannot be maximal.

Proof. Let $G$ be a chemical tree and $x \in V(G)$ be the only vertex such that $d_{x}=3$. Let $N(x)=\{a, b, c\}$. There two cases.

Case 1: Let $G$ contain a vertex which is of degree 4 . Notice that reverse degrees of all pendant vertex are 4 , and the reverse degree of the vertex $x, c_{x}=2$.

Let $l \in V(G)$ be a pendant vertex, $N(l)=\{m\}$ and let the vertex $m$ be not adjacent to the vertices $x, a, b, c$. Let $G^{\prime}$ be a chemical tree which is obtained from $G$, so that the vertex $c$ is detached from $x$ and is attached to $l$. Notice that $c_{x}=3$ and $c_{l}=3$ after the transformation. Then

$$
C M_{1}^{\beta}(G)-C M_{1}^{\beta}\left(G^{\prime}\right)=-2<0 .
$$

Case 2: Let $G$ do not contain a vertex, which is of degree 4, and contain at least two vertices of degree 3. Notice that the reverse degrees of all pendant vertices are 3 and the reverse degree of the vertex $x, c_{x}=1$. Let $l \in V(G)$ be a pendant vertex, $N(l)=\{m\}$ and the vertex $m$ be not adjacent to the vertices $x, a, b, c$. Let $G^{\prime}$ be a chemical tree, which is obtained from $G$ so that the vertex $c$ is detached from $x$ and is attached to $l$. Notice that $c_{x}=2$ and $c_{l}=2$ after the transformation. Then

$$
C M_{1}^{\beta}(G)-C M_{1}^{\beta}\left(G^{\prime}\right)=-2<0 .
$$

Lemma 2.2. If $G$ is a chemical tree with at least two vertices of degree 4 , then its first reverse Zagreb beta index cannot be maximal.

Proof. Let $G$ be a chemical tree with at least two vertices, say $x$ and $y$ that have degree 4, and $N(y)=\{a, b, c, d\}$. Let $G^{\prime}$ be a chemical tree which is obtained from $G$ so that the vertex $a$ is detached from $y$ and is attached to any pendant vertex, say $l$, which is not adjacent to $x$. Notice that $c_{y}=2$ and $c_{l}=3$ after the transformation. Then

$$
C M_{1}^{\beta}(G)-C M_{1}^{\beta}\left(G^{\prime}\right)=-4<0 .
$$

Corollary 2.2.1. If $G$ is a chemical tree, which has only one vertex of degree 4 and no vertex of degree 3, then $G$ has the maximum first reverse Zagreb beta index value among all n-vertex chemical trees.

Proof. The proof is a direct consequence of Lemma 2.1 and Lemma 2.2. 
Corollary 2.2.2. Let $G$ be an n-vertex chemical tree. Then

$$
C M_{1}^{\beta}(G) \leq 6 n-10 .
$$

Equality is attained if and only if $G$ is a chemical tree, which has only one vertex of degree 4 and no vertex of degree 3.

Proof. Let $G$ be a chemical tree, which has only one vertex of degree 4 and no vertex of degree 3 . Then $G$ has 4 pendant vertices, $n-5$ vertices of degree two and only one vertex of degree 4 . Therefore, $G$ has 4 vertices of reverse degree $4, n-5$ vertices of reverse degree 3 and only one vertex of reverse degree 1 . From the definiton of the first reverse Zagreb beta index,

$$
\left.C M_{1}^{\beta}(G)=4.4+6(n-5)+4\right)=6 n-10 .
$$

The other cases follow from Lemma 2.1 and Lemma 2.2.

\section{Conclusion}

Reverse Zagreb indices have been recently defined. In this study, we determine extremal chemical trees with respect to the first reverse Zagreb beta index. It can be interesting to find extremal unicyclic, bicyclic, tricyclic graphs of the reverse Zagreb indices. It can also be interesting to compute the exact values of the reverse Zagreb indices of some chemical structures such as nanotubes and nanotori.

\section{References}

[1] Das, K. C., \& Gutman, I. (2004) Some properties of the second Zagreb index, MATCH Commun. Math. Comput. Chem., 52, 103-112.

[2] Ediz, S. (2015) Maximum chemical trees of the second reverse Zagreb index, Pacific Journal of Applied Mathematics, 7, 291-295.

[3] Ediz, S. (2017) Extremal Chemical Trees of the First Reverse Zagreb Alpha Index, Mathematics Letters, 3, 46-49.

[4] Ediz, S. (2018) Maximal graphs of the first reverse Zagreb beta index, TWMS J. of Apl. Eng. Math., 8, 306-310.

[5] Ediz, S., \& Cancan, M. (2016) Reverse Zagreb indices of Cartesian product of graphs, International Journal of Mathematics and Computer Science, 11, 51-58.

[6] Gutman, I., \& Das, K. C. (2004) The first Zagreb index 30 years after, MATCH Commun. Math. Comput. Chem., 50, 83-92. 
[7] Gutman, I., Ruščić, B., Trinajstić, N. , \& Wilcox, C. F. (1975) Graph theory and molecular orbitals, XII. Acyclic polyenes, J. Chem. Phys., 62, 3399-3405.

[8] Gutman, I., \& Trinajstić, N. (1971) Graph theory and molecular orbitals. Total $\pi$-electron energy of alternant hydrocarbons, Chem. Phys. Lett., 17, 535-538.

[9] Nikolić, S., Kovačević, G., Miličević, A., \& Trinajstić, N. (2003) The Zagreb indices 30 years after, Croat. Chem. Acta, 76, 113-124.

[10] Stevanović, S. (2011) On the relation between the Zagreb indices, Croat. Chem. Acta, 84, $17-19$.

[11] Wiener, H. (1947) Structural determination of parafin boiling points, J. Am. Chem. Soc., 69, 17-20. 